\title{
Resonant enhancement of high-order optical nonlinearities based on atomic coherence
}

\author{
M. S. Zubairy, ${ }^{1,2}$ A. B. Matsko, ${ }^{1}$ and M. O. Scully ${ }^{1,3}$ \\ ${ }^{1}$ Department of Physics and Institute for Quantum Studies, Texas A\&M University, College Station, Texas 77843-4242 \\ ${ }^{2}$ Department of Electronics, Quaid-i-Azam University, Islamabad, Pakistan \\ ${ }^{3}$ Max-Planck-Institut für Quantenoptik, D-85748 Garching, Germany
}

(Received 15 November 2001; published 14 March 2002)

\begin{abstract}
We show that the effect of coherent population trapping may result in resonant enhancement of $\chi^{(5)}$ or higher-order nonlinearities. The enhancement is accompanied by suppression of the other linear and nonlinear susceptibility terms. This effect has promise for a realistic scheme of photon phase gates necessary for practical implementation of quantum processing protocols.
\end{abstract}

DOI: 10.1103/PhysRevA.65.043804 PACS number(s): 42.50.Gy, 03.67.-a, 42.50.Dv, 42.65.An

\section{INTRODUCTION}

It is well known that the potential devices for quantum computing and quantum information processing require unitary operations. The main problem faced in the design of such devices is the loss in quantum circuits and decoherence associated with it $[1,2]$. While the resonant two-level media can provide the highest values of nonlinearities necessary for the interaction of single optical quanta, there is an unavoidable trade-off between the values of the nonlinear susceptibility and the absorption [3]. This makes the usage of such media extremely difficult for quantum computing.

Coherent effects such as electromagnetically induced transparency (EIT) [4] and coherent population trapping (CPT) $[5,6]$ attract a lot of attention because of their ability to suppress a linear absorption of a resonant multilevel media, keeping nonlinear susceptibility at a very high level $[7,8]$. A number of studies were focused on the creation of large $\chi^{(3)}$ nonlinearities that allows either effective selfaction of an electromagnetic field on a single photon energy level [9], or effective interaction of two electromagnetic fields due to refractive $[8,10,11]$ and absorptive [12] Kerr nonlinearities.

In this paper we discuss the possibilities of resonant enhancement of $\chi^{(5)}$ and higher-order nonlinearities keeping the losses at a low level. Our proposition is based on the existence of CPT in multilevel media and resembles the early ideas of Kerr nonlinearity enhancement. Our estimations show that $\chi^{(5)}$ nonlinearity may be so high that three-photon phase gates (as described below) become feasible. Moreover, constructing an absorptive $\chi^{(5)}$ nonlinearity, we are able to remove exactly one photon each from the three modes that can be useful for quantum logic operation with photonic entangled states.

CPT results in trapping all the population of a resonant system into the so-called "dark state," which is uncoupled from the electromagnetic fields. A weak external disturbance of the dark state by means of another electromagnetic field results in the partial destruction of the dark state. This is accompanied by residual refraction and absorption that can possess nonlinear properties of great importance for quantum and nonlinear optics.

\section{MODEL}

We consider a medium (atomic, molecular, semiconductor) with the energy level structure shown in Fig. 1, where levels $\left|a_{i}\right\rangle$ have fast natural decay $\gamma_{i}$. Ground-state levels $\left|b_{i}\right\rangle$ have no decay. The coherence between levels $\left|b_{i}\right\rangle$ and $\left|b_{j}\right\rangle(i \neq j)$ has slow homogeneous decay $\gamma_{i j}$. This decay results from either finite time of flight of atoms through the interaction region (for atomic vapors) or from phononassociated decoherence (for solids). For the sake of simplicity we assume that $\gamma_{i j}=\gamma_{0}$.

The energy levels are coupled by weak probe electromagnetic fields having Rabi frequencies $\alpha_{i}$ and strong coupling fields having Rabi frequencies $\Omega_{i}\left(\left|\Omega_{i}\right| \gg\left|\alpha_{i}\right|\right)$. All the fields are resonant with associated transitions except the probe field $\alpha_{m-1}$, which has a detuning $\Delta$. The relation between Rabi frequencies of the probe fields and quantum operators describing the corresponding field mode can be written as

$$
\hat{\alpha}_{i}=\sqrt{\frac{2 \pi \wp_{i}^{2} \nu_{i}}{\hbar V_{i}}} \hat{a}_{i}=\xi_{i} \hat{a}_{i}
$$

where $\wp_{i}$ is the dipole moment of the transition $\left|a_{i}\right\rangle$ $\rightarrow\left|b_{i}\right\rangle, \nu_{i}$ is the field frequency, $V_{i}$ is the quantization volume of the mode, and $\hat{a}_{i}$ and $\hat{a}_{i}^{\dagger}$ are the annihilation and creation operators.

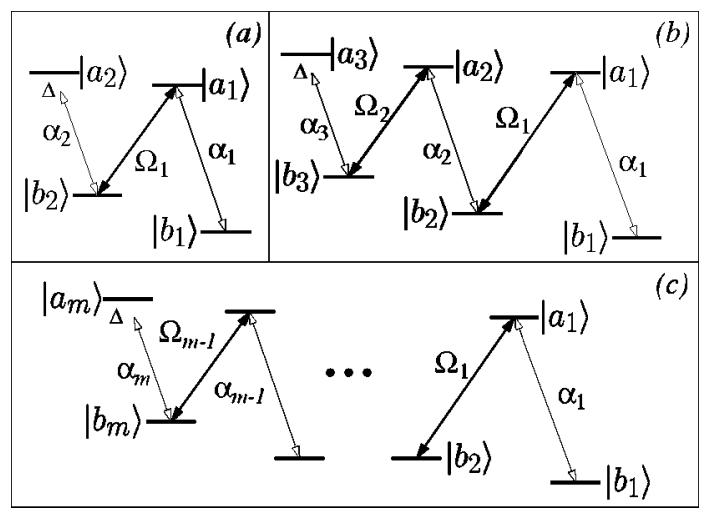

FIG. 1. Energy level schemes for the resonant enhancement of nonlinear susceptibilities of the media: (a) $\chi^{(3)}$ nonlinearity; (b) $\chi^{(5)}$ nonlinearity; (c) $\chi^{(m)}$ nonlinearity. 
The Hamiltonian of the generalized atomic system [Fig. $1(\mathrm{c})]$ in slowly varying amplitude and phase approximations is

$$
\begin{aligned}
\hat{H}_{m}= & \hbar \Delta\left|a_{m}\right\rangle\left\langle a_{m}\right|+\hbar\left(\hat{\alpha}_{m}\left|a_{m}\right\rangle\left\langle b_{m}\right|+\text { H.c. }\right)+\hbar \sum_{j=1}^{m-1}\left(\hat{\alpha}_{j}\left|a_{j}\right\rangle\right. \\
& \left.\times\left\langle b_{j}\left|+\Omega_{j}\right| a_{j}\right\rangle\left\langle b_{j+1}\right|+\text { H.c. }\right),
\end{aligned}
$$

where H.c. means Hermitian conjugate.

First we assume that $\Delta \rightarrow \infty$. Then each system, shown in Fig. 1, has a dark state. The eigenvalue of the Hamiltonian for this state is equal to zero, $\lambda_{D_{m}}=0$, i.e., $\hat{H}_{m}\left|D_{m}\right\rangle$ $=\lambda_{D_{m}}\left|D_{m}\right\rangle=0$. For the scheme shown in Fig. 1(a), the dark state is

$$
\left|D_{2}\right\rangle=\frac{\xi_{1} \sqrt{n_{1}+1}\left|b_{2}, n_{1}\right\rangle-\Omega_{1}\left|b_{1}, n_{1}+1\right\rangle}{\sqrt{\xi_{1}^{2}\left(n_{1}+1\right)+\left|\Omega_{1}\right|^{2}}} .
$$

Similarly, the dark state for the scheme shown in Fig. 1(b) is given by

$$
\begin{aligned}
\left|D_{3}\right\rangle= & \left(\xi_{1} \xi_{2} \sqrt{\left(n_{1}+1\right)\left(n_{2}+1\right)}\left|b_{3}, n_{1}, n_{2}\right\rangle-\Omega_{2} \xi_{1} \sqrt{n_{1}+1}\left|b_{2}, n_{1}, n_{2}+1\right\rangle+\Omega_{1} \Omega_{2}\left|b_{1}, n_{1}+1, n_{2}+1\right\rangle\right) / \\
& \sqrt{\xi_{1}^{2} \xi_{2}^{2}\left(n_{1}+1\right)\left(n_{2}+1\right)+\left|\Omega_{2}\right|^{2} \xi_{1}^{2}\left(n_{1}+1\right)+\left|\Omega_{1}\right|^{2}\left|\Omega_{2}\right|^{2}},
\end{aligned}
$$

and so on. In Eqs. (3) and (4), the Fock states $\left|n_{i}\right\rangle(i=1,2)$ is the eigenstate of $a_{i}^{\dagger} a_{i}$ with eigenvalues $n_{i}$.

Next we consider the case of finite $\Delta$. Strictly speaking, there is no dark state in the system in this case. However, when the detuning is large such that $\Delta \gg\left|\Omega_{j}\right| \gg|\xi|\left(n_{j}+1\right)$, $\left|b_{1}\right\rangle$ is a quasidark state corresponding to the eigenvalue that goes to zero when the detuning is infinite. In the Appendix we discuss the level scheme of Fig. 1(a) $(m=2)$ and derive the eigenstates and the eigenvectors of the Hamilonian (2). An effective Hamiltonian can then be derived in terms of the resulting $\chi^{(3)}$ nonlinearity.

Following the approach of the Appendix we can consider the case for $m=3$ [see Fig. 1(b)]. As, in the case of $m=2$, we write the Hamiltonian (2) in terms of the basis of states

$$
\begin{aligned}
& \left|b_{3}, n_{1}, n_{2}, n_{3}+1\right\rangle, \quad\left|b_{2}, n_{1}, n_{2}+1, n_{3}+1\right\rangle, \\
& \left|b_{1}, n_{1}+1, n_{2}+1, n_{3}+1\right\rangle, \quad\left|a_{3}, n_{1}, n_{2}, n_{3}\right\rangle, \\
& \left|a_{2}, n_{1}, n_{2}, n_{3}+1\right\rangle, \quad\left|a_{1}, n_{1}, n_{2}+1, n_{3}+1\right\rangle .
\end{aligned}
$$

The corresponding equation for the eigenvalues is then of the form

$$
\begin{gathered}
\lambda^{6}+a \lambda^{5}+b \lambda^{4}+c \lambda^{3}+d \lambda^{2}+e \lambda+f=0, \\
a=\Delta, \quad f=-\left|\alpha_{1}\right|^{2}\left|\alpha_{2}\right|^{2}\left|\alpha_{3}\right|^{2}, \\
b=-\left|\alpha_{1}\right|^{2}-\left|\Omega_{1}\right|^{2}-\left|\alpha_{2}\right|^{2}-\left|\Omega_{2}\right|^{2}-\left|\alpha_{3}\right|^{2}, \\
c=\Delta\left(\left|\Omega_{1}\right|^{2}+\left|\Omega_{2}\right|^{2}+\left|\alpha_{3}\right|^{2}\right), \\
d=\left|\alpha_{1}\right|^{2}\left|\alpha_{2}\right|^{2}+\left|\alpha_{2}\right|^{2}\left|\alpha_{3}\right|^{2}+\left|\alpha_{1}\right|^{2}\left|\alpha_{3}\right|^{2}+\left|\Omega_{1}\right|^{2}\left|\alpha_{3}\right|^{2} \\
+\left|\Omega_{2}\right|^{2}\left|\Omega_{2}\right|^{2}+\left|\Omega_{2}\right|^{2}\left|\alpha_{1}\right|^{2}, \\
e=-\Delta\left(\left|\alpha_{1}\right|^{2}\left|\alpha_{3}\right|^{2}+\left|\Omega_{2}\right|^{2}\left|\alpha_{1}\right|^{2}-\left|\Omega_{2}\right|^{2}\left|\Omega_{2}\right|^{2}\right),
\end{gathered}
$$

where $\lambda_{k}$ are the eigenvalues [roots of Eq. (5)] and $\left|\lambda_{k}\right\rangle$ are the corresponding eigenvectors. Here the eigenvalue for the disturbed dark state is

$$
\lambda_{6}=\lambda_{\tilde{D}_{3}} \approx \frac{\xi_{3}^{2}\left(n_{3}+1\right)}{\Delta} \frac{\xi_{2}^{2}\left(n_{2}+1\right)}{\left|\Omega_{2}\right|^{2}} \frac{\xi_{1}^{2}\left(n_{1}+1\right)}{\left|\Omega_{1}\right|^{2}},
$$

and the corresponding eigenstate is

$$
\left|\widetilde{D}_{3}\right\rangle=\left|b_{1}, n_{1}+1, n_{2}+1, n_{3}+1\right\rangle .
$$

It can be shown that none of the other eigenstaes $\left|\lambda_{j}\right\rangle$ ( $j$ $=1-5)$ contain the state $\left|b_{1}\right\rangle$. Thus for atoms initially in the state $\left|b_{1}\right\rangle$, the approximate Hamiltonian is

$$
\begin{aligned}
\hat{H} \approx & \hbar \sum_{n_{1}, n_{2}, n_{3}}^{\infty} \frac{\xi_{3}^{2}\left(n_{3}+1\right)}{\Delta} \frac{\xi_{2}^{2}\left(n_{2}+1\right)}{\left|\Omega_{2}\right|^{2}} \frac{\xi_{1}^{2}\left(n_{1}+1\right)}{\left|\Omega_{1}\right|^{2}} \\
& \times\left|b_{1}, n_{1}+1, n_{2}+1, n_{3}+1\right\rangle \\
& \times\left\langle b_{1}, n_{1}+1, n_{2}+1, n_{3}+1\right| .
\end{aligned}
$$

To derive an effective Hamiltonian from Eq. (6), we exchange $n_{k}+1$ by the photon number operator $\hat{a}_{k}^{\dagger} \hat{a}_{k}$.

We can derive now an effective Hamiltonian for the interaction of the probe fields with a single atom in the general form

$$
\hat{H}_{m}=(-1)^{m+1} \hbar \frac{\xi_{m}^{2} \hat{a}_{m}^{\dagger} \hat{a}_{m}^{m-1}}{\Delta} \prod_{j=1}^{m} \frac{\xi_{j}^{2} \hat{a}_{j}^{\dagger} \hat{a}_{j}}{\left|\Omega_{j}\right|^{2}} .
$$


As all the atomic population is eventually optically pumped into the dark state and, as discussed above, almost all the population of the dark state is in the $\left|b_{1}\right\rangle$ state, this interaction Hamiltonian does not contain atomic operators.

We next estimate the maximum strength of the field interaction in the case when the medium shows the enhanced $\chi^{(5)}$ nonlinearity (Fig. 1), i.e.,

$$
\hat{H}_{3}=\hbar \widetilde{\delta} \hat{a}_{1}^{\dagger} \hat{a}_{1} \hat{a}_{2}^{\dagger} \hat{a}_{2} \hat{a}_{3}^{\dagger} \hat{a}_{3},
$$

where $\widetilde{\delta}$ is the coupling constant. Assuming that all parameters of the optical transitions are nearly the same and by using the expression $\wp^{2} / \hbar=3 c^{3} \gamma / 4 \nu^{3}$, we get

$$
\widetilde{\delta}=\frac{3}{8 \pi} N \lambda^{2} c \frac{\gamma}{\Delta} \frac{1}{n_{\Omega 1} n_{\Omega 2}},
$$

where $N$ is the atomic density in the interaction volume, $\lambda$ is the optical wavelength, $c$ is the speed of light in the vacuum, and $n_{\Omega} \gg 1$ is the average number of the photons of the coupling field in the interaction region. We assume here that the interaction and quantization volumes are the same.

The minimum value of the photon number in the coupling field $n_{\Omega}$ is restricted by the necessary condition $|\Omega| \gg \sqrt{\gamma_{0} \gamma}$ of the validity of the CPT approximation. For an atomic trap, $\gamma_{0} \simeq 10^{-5} \gamma \approx 50 \mathrm{~Hz}$ can be reached, and $|\Omega|^{2}$ should exceed $10^{-5} \gamma^{2}$ which corresponds to the intensity of $0.1 \mu \mathrm{W} / \mathrm{cm}^{2}$.

We now estimate the coupling constant $\widetilde{\delta}$. For the linear size of the interaction region $L \simeq 0.3 \mathrm{~cm}$, one can have the power of the coupling field $P_{\Omega} \simeq 0.3 \mu \mathrm{W}$, so that $1 /\left(n_{\Omega 1} n_{\Omega 2}\right) \approx 0.01$ (for the interaction time $\sim L / c$ ). Taking $N \simeq 310^{12} \mathrm{~cm}^{-3}, \lambda \simeq 10^{-4} \mathrm{~cm}$, and $\gamma / \Delta=0.3$, we get $\widetilde{\delta}$ $\approx 310^{11} \mathrm{~s}^{-1}$. If we assume that the interaction time is equal to $L / c$, we get phase shift $\widetilde{\delta} \tau \approx 3 \mathrm{rad}$.

Therefore, atomic coherence allows us to achieve a strong nonlinear interaction among three electromagnetic waves. This nonlinearity can be only a couple of orders of magnitude less than the maximum susceptibility of an atomic system. We point out that a single photon absorption of a weak resonant radiation propagating through a two-level system, which actually determines the maximum of the susceptibility (inverse Beer's length), is equal to $3 N \lambda^{2} / 8 \pi$ [cf. Eq. (12)]. However, winning in the value of the nonlinearity we lose in the bandwidth of the nonlinearity. The effective interaction can be achieved for the continuous waves only. An experimental realization of the interaction for light pulses is not so simple. We discuss this problem in the next section.

If the detuning $\Delta$ is small compared to $\gamma$, the refractive nonlinearity is small. However, the absorptive nonlinearity is large. In the case of the scheme Fig. 1(a) the probability of simultaneous absorption of two photons from $\alpha_{1}$ and $\alpha_{2}$ fields significantly exceed the probability of independent photon absorption [12]. In the more general case considered here [Fig. 1(b)], the probability of three-photon absorption significantly exceeds the other absorption probabilities.

If initially only the driving fields $\Omega_{1}$ and $\Omega_{2}$ are applied to the atom, only level $\left|b_{1}\right\rangle$ is populated. When the atom starts to interact with probe photons there is no absorption unless $\alpha_{1}=0$. If $\alpha_{1} \neq 0$, and $\alpha_{2}=\alpha_{3}=0$ there is no absorption due to EIT. The same is true if $\alpha_{1} \neq 0$ and $\alpha_{2} \neq 0$, but $\alpha_{3}=0$. Only when all three probe fields are present the absorption is possible.

The probability of spontaneous photon emission per unit time per single atom shown in Fig. 1(b) can be estimated as

$$
W_{m}=\gamma \sum_{j=1}^{m}\left\langle\widetilde{D}_{m} \mid a_{j}\right\rangle\left\langle a_{j} \mid \widetilde{D}_{m}\right\rangle .
$$

In the approximation of strong drive and weak probe fields we find

$$
W_{m} \simeq \frac{\xi_{m}^{2} n_{m}^{m-1}}{\gamma} \prod_{j=1}^{m} \frac{\xi_{j}^{2} n_{j}}{\left|\Omega_{j}\right|^{2}}
$$

The probability of $m$ photon absorption during interaction time $\tau$ is equal to $W_{m} \tau$ and can be very high. We can simply send the atoms through a cavity with excited probe modes to remove three photons or put the absorbing material inside the cavity.

Let us discuss the possibilities of experimental implementation of the schemes. For the simplest case of two-photon refraction and absorption [scheme in Fig. 1(a)] an appropriate choice is the $D_{1}$ line of ${ }^{87} \mathrm{Rb}$ [12]. For the level scheme shown in Fig. 1(b) any material that has a triplet in the Ground-state and not less than a triplet in the excited state would be appropriate. An example is the $\mathrm{Pr}^{3+}$-doped $\mathrm{Y}_{2} \mathrm{SiO}_{5}$ (Pr:YSO) crystal, used recently to demonstrate a slow group velocity of light [13].

It is worth mentioning here that we have so far considered a homogeneously broadened medium and a realization of the scheme in a Doppler-broadened medium is difficult. The detuning $\Delta$ should be large enough to exceed the Doppler width $\Delta_{d}$. The coupling fields should be much stronger too. The usual condition to have a Doppler-free EIT is $|\Omega|$ $\gg \Delta_{d} \sqrt{\gamma_{0} / \gamma}$. If these conditions are satisfied the factor $\widetilde{\delta} \tau$ becomes 5-6 orders of magnitude less then the factor calculated above.

\section{PROPAGATION PROBLEM}

The above analysis is valid for the $\mathrm{cw}$ regime. Propagation substantially modifies the nonlinear interaction, including both the refractive and absorptive nonlinearities. The reason for this modification was recognized in [10]. Let us present here a simple argument to explain the phenomenon and estimate a phase shift occurring among copropagating pulses in the nonlinear medium.

We consider here the probe pulses initially having the same shape and overlapping envelops. This picture changes after the pulses enter the nonlinear medium. As almost all the atomic population is collected in the $\left|b_{1}\right\rangle$ atomic level (see Fig. 1), the pulse of the probe field $\alpha_{1}$ propagates much slower than the other pulses [14]. The group velocity of this pulse can be estimated as 


$$
v_{g 1} \simeq\left[\frac{3}{8 \pi} N \lambda^{2} \frac{\gamma}{\left|\Omega_{1}\right|^{2}}\right]^{-1} \ll c .
$$

The spatial dimensions of the pulse changes, too. If the length of the pulse in the vacuum was $L_{\alpha}$, it reduces up to $L_{\alpha} v_{g 1} / c$ in the medium. The peak Rabi frequency of the pulse stays unchanged.

The other probe pulses propagate with the speed close to the speed of light in the vacuum because the atomic states the pulses interact with are almost empty. These pulses pass the slow pulse and leave the medium first.

To estimate the phase shift acquired by the probe pulses due to the interaction using Hamiltonian (10), we have to know the interaction time and the number of atoms interacting with the pulses during this time. We consider the case when the length of the medium exceeds the compressed length of the slow pulse, i.e., $L \gg L_{\alpha} v_{g 1} / c$. Then the interaction time is determined by the passage time of the fast pulses through the slow one. This time is equal to $L_{\alpha} / c$. The number of atoms interacting with the pulses is determined by the volume of the spatially compressed slow pulse and is much smaller than the number of atoms contained in the cell. We can estimate this number as $N L_{\alpha} \mathcal{A} v_{g 1} / c$, where $\mathcal{A}$ is the cross-sectional area of the probe laser beams.

We now estimate the phase shift in the case of interaction of three pulses. The effective interaction Hamiltonian can be presented in the form similar to Eq. (11),

$$
\hat{H}_{3}=\hbar \widetilde{\delta}^{\prime} \hat{a}_{1}^{\dagger} \hat{a}_{1} \hat{a}_{2}^{\dagger} \hat{a}_{2} \hat{a}_{3}^{\dagger} \hat{a}_{3},
$$

where $\widetilde{\delta}^{\prime}$ is the new coupling constant

$$
\widetilde{\delta}^{\prime}=\frac{3}{8 \pi} \frac{\gamma}{\Delta} \frac{\lambda^{2}}{\mathcal{A}} \frac{1}{n_{\Omega 2}} \frac{c}{L_{\alpha}} .
$$

This coupling constant is $L c /\left(L_{\alpha} v_{g 1}\right) \gg 1$ times weaker than the coupling constant $\widetilde{\delta}(12)$ derived in cw approximation. Our estimation gives the same result as the strict calculation performed for the case of the two probe fields in [10]. The numerical factor " $3 /(8 \pi)$ " can be modified depending on the pulse shapes.

According to Eq. (17) it is impossible to get a phase shift per probe photon more than unity because the maximum phase shift $\widetilde{\delta}^{\prime} L_{\alpha} / c$ contains all multipliers less than 1 . To overcome this situation, the technique proposed in [11] may be applied. The main idea is to slow down all the probe pulses so that their group velocities are equal to each other. Then the interaction time between the pulses increases by the factor $L c /\left(L_{\alpha} v_{g 1}\right)$, thus returning us to our previous $\mathrm{cw}$ result.

As it was noted in [11], group velocity of the fast pulse in an $N$-type level scheme can be reduced if the atomic cell contains not only the atoms that induce the nonlinear interaction between electromagnetic fields, but also other atoms. These atoms create a $\Lambda$-type scheme for the fast pulse and slow it down [14]. By choosing an appropriate density of the miscellaneous gas we are able to match the group velocities and increase the nonlinear interaction. The $\Lambda$-type atoms do not introduce nonlinearity itself and do not change the nonlinear interaction we discussed above.

Slowing down of the light pulse can be achieved by using different isotopes in the atomic cell. One isotope creates an effective nonlinear interaction between fields while the other isotope influences on the group velocity of the pulses.

The idea of controlling the group velocity of the pulses by means of different atoms works for us, too. However, it might be impractical for the case of many fields. Instead of real atoms quantum dots ("artificial atoms") may be used. We can control the level structure of a quantum dot by changing its shape and size. Practical usage of quantum dots, however, is complicated because of different types of nonradiative broadenings associated with quantum dots [15].

Another problem is associated with the nonlinear pulse propagation. Due to the nonlinear interaction, different parts of the pulse acquires different phase shift during propagation. For the case of a single-mode (plane wave) field discussed so far, this is not a problem. We first explain the essence of the problem considering the example of two pulses interacting via atoms in $N$-type level configuration [Fig. 1(a)].

If the pulses move with the same velocity $v_{g}$, the solution at the cell exit can be written in the form [11]

$$
\hat{E}_{\alpha 1}(L, t)=\hat{E}_{\alpha 1}\left(0, t^{\prime}\right) \exp \left[\operatorname{ig} L \hat{E}_{\alpha 2}^{\dagger}\left(0, t^{\prime}\right) \hat{E}_{\alpha 2}\left(0, t^{\prime}\right)\right]
$$

$$
\hat{E}_{\alpha 2}(L, t)=\hat{E}_{\alpha 2}\left(0, t^{\prime}\right) \exp \left[\operatorname{ig} L \hat{E}_{\alpha 1}^{\dagger}\left(0, t^{\prime}\right) \hat{E}_{\alpha 1}\left(0, t^{\prime}\right)\right]
$$

where $\hat{E}_{\alpha 1}$ and $\hat{E}_{\alpha 2}$ are the quantum operators of pulses' field, $t^{\prime}=t-L / v_{g}$ is the retarded time, and $g$ is the interaction constant. As it follows from Eqs. (18) and (19) the nonlinear phase shift changes for each pulse with time. This shift is small in the tails of the pulse and large in the pulse center. This can restrict the application of the nonlinearity significantly.

The problem of different phase shifts is valid for all kinds of nonlinearities because it depends on the pulse geometry. We see two ways for the solution of this problem. One way is based on the obvious use of long pulses with small tails. The inhomogeneous phase shift appears on the tails only and, hence, it can be reduced. Another way is based on the usage of pulses with a little mismatch between group velocities. If we send a slower pulse on the front of a faster pulse, the faster pulse passes the slower pulse in the nonlinear medium. During this passage each point of one pulse interacts with each point of the other pulse. Therefore, each point of both the pulses acquires the same, averaged, phase shift. The group velocity mismatch should be chosen in such a way that the pulse interaction time nearly corresponds to the time of pulse crossing the nonlinear medium [16]. It might be a problem, however, to use this technique to achieve efficient interaction between multiple (three or more) pulses. This question will be discussed in detail elsewhere. 


\section{MULTIPHOTON QUANTUM PHASE GATES}

We now discuss the potential applications of the logic gates based on the interaction (11). It is well known that a unitary transformation (one-bit gate) and a two-bit conditional quantum phase gate form a universal quantum computer. The one-bit quantum gate for the $i^{t h}$ qubit is given by

$$
U_{\theta, \phi}^{i}=\left(\begin{array}{cc}
\cos \theta & -i e^{-i \phi} \sin \theta \\
-i e^{i \phi} \sin \theta & \cos \theta
\end{array}\right) .
$$

The transformation for a two-bit quantum phase gate is given by

$$
Q_{\eta}^{(2)}\left|\alpha_{1}, \alpha_{2}\right\rangle=\exp \left(i \eta \delta_{\alpha_{1}, 1} \delta_{\alpha_{2}, 1}\right)\left|\alpha_{1}, \alpha_{2}\right\rangle,
$$

where $\left|\alpha_{1}\right\rangle$ and $\left|\alpha_{2}\right\rangle$ stand for the basis states $|0\rangle$ or $|1\rangle$ of the two qubits, and $\delta_{\alpha_{1}, 1}$ is the Kronecker $\delta$ symbol, which is equal to 1 if there is a photon in the mode $\alpha_{1}$ and zero otherwise. Thus the quantum phase gate introduces a phase $\eta$ only when both the qubits in the input state are 1 . Thus, for example, a representation of the quantum phase gate with $\eta=\pi$ is given by

$$
\begin{aligned}
Q_{\pi}^{(2)}= & |0,0\rangle\langle 0,0|+| 0,1\rangle\langle 0,1| \\
& +|1,0\rangle\langle 1,0|-| 1,1\rangle\langle 1,1| .
\end{aligned}
$$

This phase gate can be realized by means of a lossless Kerr medium, for example using the medium with levels shown in Fig. 1(a), i.e., $Q^{(2)}=\exp \left(-i \hat{H}_{2} \tau / \hbar\right)$.

There are, however, situations where one may need $m$-bit quantum phase gate defined via

$$
\begin{aligned}
& Q_{\eta}^{(m)}\left|\alpha_{1}, \alpha_{2}, \ldots \alpha_{m}\right\rangle \\
& \quad=\exp \left(i \eta \delta_{\alpha_{1}, 1} \delta_{\alpha_{2}, 1} \ldots \delta_{\alpha_{m, 1}}\right)\left|\alpha_{1}, \alpha_{2}, \ldots \alpha_{m}\right\rangle,
\end{aligned}
$$

i.e., a phase $\eta$ is introduced when all the qubits are in state $|1\rangle$. It is easily seen that such a multiphoton phase gate can be constructed via interaction (10) $Q_{\eta}^{(m)}=\exp \left(-i \hat{H}_{m} \tau / \hbar\right), \eta$ $=\widetilde{\delta} \tau$. Such a construction is extremely simple as compared to a possible but cumbersome implementation via one-bit unitary gate and two-bit quantum phase gate. For example, an implementation of one three-bit quantum phase gate may require five two-bit quantum phase gates and four one-bit unitary gate [17]. Such higher-order quantum phase gates may have important applications in quantum computing. Here we discuss one such application in the implementation of Grover's quantum search algorithm [18].

The Grover's algorithm for the search of an item in an unsorted database containing $N=2^{q}$ items can be accomplished as follows. In the first step, an initial state $\left|0_{1}, 0_{2}, \ldots, 0_{q}\right\rangle$ undergoes Walsh-Hadamard transformation $\mathcal{W}$ which rotates each qubit from $|0\rangle$ to $(|0\rangle+|1\rangle) / \sqrt{2}$ and $|1\rangle$ to $(|1\rangle-|0\rangle) / \sqrt{2}$. The resulting state is

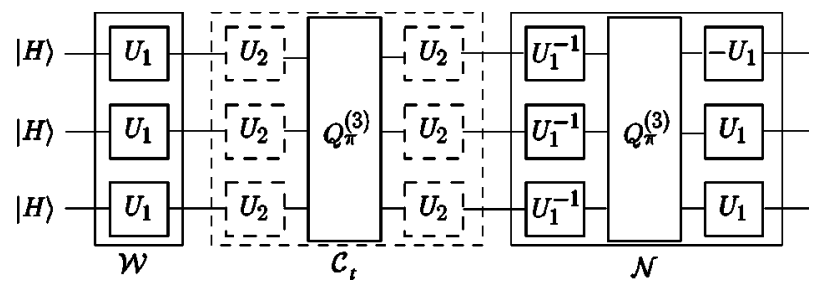

FIG. 2. A scheme for the implementation of Grover's algorithm for eight objects $(q=3)$ based on the multiphoton quantum phase gate.

$$
|s\rangle=\frac{1}{\sqrt{N}} \sum_{a_{1}, a_{2}, \ldots, a_{q}=0}^{1}\left|a_{1}, a_{2}, \ldots, a_{q}\right\rangle,
$$

i.e., a superposition of all possible $N$ states. Now an oracle flips the phase of a target state $|t\rangle$ in the superposition $|s\rangle$ via an operation $\mathcal{C}_{t}=1-2|t\rangle\langle t|$. The objective is to determine the target state. Next an inversion about the mean is accomplished via $\mathcal{N}=1-2|s\rangle\langle s|$. In the Grover's algorithm the combined operator $\mathcal{G}=-\mathcal{C}_{t} \mathcal{N}$ is applied on the initial state $k$ times. When $k=\pi \sqrt{ } N / 4$, the probability of recovering the target state $|t\rangle$ becomes maximum [19].

In Fig. 2 we present a scheme for the implementation of Grover's algorithm for eight objects $(q=3)$ based on the multiphoton quantum phase gate discussed earlier. The basis of the quantum states we are working in can consist of the horizontally and vertically polarized one-photon states

$$
|H\rangle=\left(\begin{array}{l}
1 \\
0
\end{array}\right), \quad|V\rangle=\left(\begin{array}{l}
0 \\
1
\end{array}\right) .
$$

Then the unitary transformations

$$
U_{1}=\frac{1}{\sqrt{2}}\left(\begin{array}{cc}
1 & -1 \\
1 & 1
\end{array}\right), \quad U_{2}=\left(\begin{array}{ll}
0 & 1 \\
1 & 0
\end{array}\right)
$$

can be realized by a $\lambda / 2$ plate rotated at a $\pi / 8$ angle and a $\lambda / 2$ plate rotated at a $\pi / 4$ angle.

\section{CONCLUSION}

In conclusion, we have proposed a realization of media with resonantly enhanced $\chi^{(5)}$ and higher orders of optical nonlinearity where one-photon resonant absorption is suppressed due to coherence effects. Such media are useful for the creation of logical elements necessary for quantum computation. We have discussed an example of Grover's search algorithm improved by the application of nonlinear gates based on the nonlinearities of higher orders.

\section{ACKNOWLEDGMENTS}

The authors gratefully acknowledge the support from Air Force Research Laboratories (Rome, New York), DARPAQuIST, TAMU Telecommunication and Informatics Task Force (TITF) initiative, the Office of Naval Research, and the Welch Foundation. 


\section{APPENDIX: DERIVATION OF AN EFFECTIVE HAMILTONIAN FOR A FOUR-LEVEL $N$ SCHEME}

Here we consider the level scheme shown in Fig. 1(a). The Hamiltonian describing the atom-field interaction has the form [cf. Eq. (2)]

$$
\hat{H}_{2}=\hbar\left(\begin{array}{cccc}
0 & \hat{\alpha}_{1} & \Omega_{1} & 0 \\
\hat{\alpha}_{1}^{\dagger} & 0 & 0 & 0 \\
\Omega_{1}^{*} & 0 & 0 & \hat{\alpha}_{2}^{\dagger} \\
0 & 0 & \hat{\alpha}_{2} & \Delta
\end{array}\right)
$$

We introduce the basis of states

$$
\begin{array}{cc}
\left|b_{1}, n_{1}+1, n_{2}+1\right\rangle, & \left|a_{1}, n_{1}, n_{2}+1\right\rangle, \\
\left|b_{2}, n_{1}, n_{2}+1\right\rangle, & \left|a_{2}, n_{1}, n_{2}\right\rangle,
\end{array}
$$

and calculate the eigenvalues of the Hamiltonian in this basis. The eigenvalue equation is

$$
\left|\begin{array}{cccc}
-\lambda & \xi_{1} \sqrt{n_{1}+1} & \Omega_{1}^{*} & 0 \\
\xi_{1} \sqrt{n_{1}+1} & -\lambda & 0 & 0 \\
\Omega_{1} & 0 & -\lambda & \xi_{2} \sqrt{n_{2}+1} \\
0 & 0 & \xi_{2} \sqrt{n_{2}+1} & \Delta-\lambda
\end{array}\right|=0 .
$$

Equation (A3) can be rewritten in the usual algebraic form

$$
\lambda^{4}+a \lambda^{3}+b \lambda^{2}+c \lambda+d=0
$$

where

$$
\begin{gathered}
a=-\Delta, d=\xi_{1}^{2} \xi_{2}^{2}\left(n_{1}+1\right)\left(n_{2}+1\right), \\
b=-\xi_{1}^{2}\left(n_{1}+1\right)-\left|\Omega_{1}\right|^{2}-\xi_{2}^{2}\left(n_{2}+1\right), \\
c=\Delta\left[\xi_{1}^{2}\left(n_{1}+1\right)+\left|\Omega_{1}\right|^{2}\right] .
\end{gathered}
$$

We solve this equation assuming $\Delta \gg\left|\Omega_{1}\right| \gg \xi_{i} \sqrt{n_{i}+1}$. The resultant eigenvalues are

$$
\begin{gathered}
\lambda_{1} \simeq \Delta, \\
\lambda_{2} \simeq \sqrt{\xi_{1}^{2}\left(n_{1}+1\right)+\left|\Omega_{1}\right|^{2}}, \\
\lambda_{3} \simeq-\sqrt{\xi_{1}^{2}\left(n_{1}+1\right)+\left|\Omega_{1}\right|^{2}}, \\
\lambda_{4} \equiv \lambda_{\widetilde{D}_{2}} \simeq-\frac{\xi_{1}^{2} \xi_{2}^{2}\left(n_{1}+1\right)\left(n_{2}+1\right)}{\Delta\left|\Omega_{1}\right|^{2}} .
\end{gathered}
$$

It is easy to calculate the corresponding eigenvectors and they are

$$
\left|\lambda_{1}\right\rangle \simeq\left|a_{2}, n_{1}, n_{2}\right\rangle,
$$

$$
\begin{gathered}
\left|\lambda_{2}\right\rangle \simeq \frac{1}{\sqrt{2}}\left(\left|a_{1}, n_{1}, n_{2}+1\right\rangle+\left|b_{2}, n_{1}, n_{2}+1\right\rangle\right), \\
\left|\lambda_{3}\right\rangle \simeq \frac{1}{\sqrt{2}}\left(\left|a_{1}, n_{1}, n_{2}+1\right\rangle-\left|b_{2}, n_{1}, n_{2}+1\right\rangle\right), \\
\left|\lambda_{4}\right\rangle \equiv\left|\widetilde{D}_{2}\right\rangle \simeq\left|b_{1}, n_{1}+1, n_{2}+1\right\rangle .
\end{gathered}
$$

The eigenvlue $\lambda_{\widetilde{D}_{2}}$ and the corresponding eigenvector $\left|\widetilde{D}_{2}\right\rangle$ correspond to the disturbed dark state. Thus an atom initially in state $|b\rangle$ will remain there and the atom-field interaction is purely dispersive as shown below. form

The Hamiltonian of the system can now be written in the

$$
\hat{H}_{2}=\sum_{k=1}^{4} \sum_{n_{1}, n_{2}}^{\infty} \hbar \lambda_{k}\left|\lambda_{k}\right\rangle\left\langle\lambda_{k}\right|,
$$

and for an atom initially in the state $|b\rangle$ can be approximated as

$$
\hat{H}_{2} \approx \sum_{n_{1}, n_{2}}^{\infty} \hbar \lambda_{\widetilde{D}_{2}}\left|\widetilde{D}_{2}\right\rangle\left\langle\widetilde{D}_{2}\right|
$$

where $\lambda_{k}$ are the eigenvalues [roots of Eq. (A4)] and $\left|\lambda_{k}\right\rangle$ are the corresponding eigenvectors. Using the above results we represent this expression as

$$
\begin{aligned}
\hat{H} \approx & -\hbar \sum_{n_{1}, n_{2}}^{\infty} \frac{\xi_{2}^{2}\left(n_{2}+1\right)}{\Delta} \frac{\xi_{1}^{2}\left(n_{1}+1\right)}{\left|\Omega_{1}\right|^{2}} \\
& \times\left|b_{1}, n_{1}+1, n_{2}+1\right\rangle\left\langle b_{1}, n_{1}+1, n_{2}+1\right| .
\end{aligned}
$$

To derive an effective Hamiltonian from Eq. (A5), we exchange $n_{k}+1$ by the photon number operator $\hat{a}_{k}^{\dagger} \hat{a}_{k}$,

$$
\begin{aligned}
\hat{H}_{2} \approx & -\hbar \frac{\xi_{1}^{2} \hat{a}_{1}^{\dagger} \hat{a}_{1}}{\Delta} \frac{\xi_{2}^{2} \hat{a}_{2}^{\dagger} \hat{a}_{2}}{\left|\Omega_{1}\right|^{2}} \sum_{n_{1}, n_{2}}^{\infty}\left|b_{1}, n_{1}+1, n_{2}+1\right\rangle \\
& \times\left\langle b_{1}, n_{1}+1, n_{2}+1\right| \\
= & -\hbar \frac{\xi_{1}^{2} \hat{a}_{1}^{\dagger} \hat{a}_{1}}{\Delta} \frac{\xi_{2}^{2} \hat{a}_{2}^{\dagger} \hat{a}_{2}}{\left|\Omega_{1}\right|^{2}} .
\end{aligned}
$$

As all the atomic population is eventually optically pumped into the dark state, almost all the population of the dark state is in the $\left|b_{1}\right\rangle$ state. Therefore, we have not included the atomic operators in the interaction Hamiltonian in the last line of Eq. (A7). To keep all the population in the dark state during the interaction process, we need to satisfy adiabaticity the condition $\Omega T \gg 1$, where $T$ is a characteristic time of the process. 
[1] A. Ekert and R. Jozsa, Rev. Mod. Phys. 68, 733 (1996).

[2] A. Steane, Rep. Prog. Phys. 61, 117 (1998).

[3] K. Watanabe and Y. Yamamoto, Phys. Rev. A 42, 1699 (1990).

[4] For reviews on EIT see S.E. Harris, Phys. Today, 50(7), 36 (1997); J.P. Marangos, J. Mod. Opt. 45, 471 (1998).

[5] For reviews on CPT see E. Arimondo, in Progress in Optics, edited by E. Wolf (Elsevier Science, Amsterdam, 1996), Vol. XXXV, p. 257.

[6] M. O. Scully and M. S. Zubairy, Quantum Optics (Cambridge University Press, Cambridge, 1997).

[7] S.E. Harris, J.E. Field, and A. Imamoglu, Phys. Rev. Lett. 64, 1107 (1990).

[8] H. Schmidt and A. Imamoglu, Opt. Lett. 21, 1936 (1996).

[9] A. Imamoglu, H. Schmidt, G. Woods, and M. Deutsch, Phys. Rev. Lett. 79, 1467 (1997); S. Rebic, S.M. Tan, A.S. Parkins, and D.F. Walls, J. Opt. B: Quantum Semiclassical Opt. 1, 490 (1999); K.M. Gheri, W. Alge, and P. Grangier, Phys. Rev. A 60, R2673 (1999); A.D. Greentree, J.A. Vaccaro, S.R. de Echaniz, A.V. Durrant, and J.P. Marangos, J. Opt. B: Quantum Semiclassical Opt. 2, 252 (2000).

[10] S.E. Harris and L.V. Hau, Phys. Rev. Lett. 82, 4611 (1999).
[11] M.D. Lukin and A. Imamoglu, Phys. Rev. Lett. 84, 1419 (2000).

[12] S.E. Harris and Y. Yamamoto, Phys. Rev. Lett. 81, 3611 (1998); B.S. Ham and P.R. Hemmer, ibid. 84, 4080 (2000); M. Yan, E.G. Rickey, and Y. Zhu, Opt. Lett. 26, 548 (2001); M. Yan, E.G. Rickey, and Y. Zhu, Phys. Rev. A 64, 041801 (2001).

[13] A.V. Turukhin, V.S. Sudarshanam, M.S. Shahriar, J.A. Musser, B.S. Hain, and P.R. Hemmer, Phys. Rev. Lett. 88, 023602 (2201).

[14] A.B. Matsko, O. Kocharovskaya, Y. Rostovtsev, G.R. Welch, A.S. Zibrov, and M.O. Scully, Adv. At., Mol., Opt. Phys. 46, 191 (2001).

[15] L. Banyai and S. W. Koch, Semiconductor Quantum Dots (World Scientific, Singapore, 1993).

[16] Similar technique is used to create efficient cross-phase modulation for solitons: H.A. Haus, K. Watanabe, and Y. Yamamoto, J. Opt. Soc. Am. B 6, 1138 (1989).

[17] Z. Diao,M. S. Zubairy, and G. Chen (unpublished).

[18] L.K. Grover, Phys. Rev. Lett. 79, 325 (1997).

[19] E. Farhi and S. Gutmann, Phys. Rev. A 57, 2403 (1998). 